Article

\title{
Acute Effect of the Compression Technique on the Electromyographic Activity of the Masticatory Muscles and Mouth Opening in Subjects with Active Myofascial Trigger Points
}

\author{
Michał Ginszt ${ }^{1, *}\left(\mathbb{0}\right.$, Grzegorz Zieliński ${ }^{2} \oplus$, Marcin Berger ${ }^{3}$, Jacek Szkutnik ${ }^{4}$, \\ Magdalena Bakalczuk ${ }^{4}$ and Piotr Majcher ${ }^{1}$ \\ 1 Department of Rehabilitation and Physiotherapy, Medical University of Lublin, 20-093 Lublin, Poland; \\ piotr.majcher@umlub.pl \\ 2 Department of Sports Medicine, Medical University of Lublin, 20-093 Lublin, Poland; \\ grzegorz.zielinski@umlub.pl \\ 3 Chair and Department of Dental Surgery, Medical University of Lublin, 20-093 Lublin, Poland; \\ marcin.berger@umlub.pl \\ 4 Department of Functional Masticatory Disorders, Medical University of Lublin, 20-093 Lublin, Poland; \\ jacek.szkutnik@umlub.pl (J.S.); magdalena.bakalczuk@umlub.pl (M.B.) \\ * Correspondence: michal.ginszt@umlub.pl; Tel.: +48-81-448-6780
}

Received: 5 October 2020; Accepted: 30 October 2020; Published: 2 November 2020

\begin{abstract}
Active myofascial trigger points (MTrPs) in masticatory muscles are associated with a reduced range of motion and muscle weakness within the stomatognathic system. However, it is hard to identify the most effective treatment technique for disorders associated with MTrPs. The objective of this study was to analyze the acute effect of the compression technique (CT) on active maximal mouth opening (MMO) and electromyographic activity of the masseter (MM) and temporalis anterior (TA) muscles in subjects with active myofascial trigger points in the MM muscles. The study group comprised 26 women (mean age $22 \pm 2$ ) with bilateral active myofascial trigger points (MTrPs) in the MM. The control group comprised 26 healthy women (mean age $22 \pm 1$ ) without the presence of MTrPs in the MM. Masticatory muscle activity was recorded in two conditions (during resting mandibular position and maximum voluntary clenching) before and after the application of the CT to the MTrPs in MM. After the CT application, a significant decrease in resting activity $(3.09 \mu \mathrm{V} v \mathrm{vs}$. $2.37 \mu \mathrm{V}, p=0.006)$ and a significant increase in clenching activity $(110.20 \mu \mathrm{V}$ vs. $139.06 \mu \mathrm{V}, p=0.014)$ within the MM muscles were observed in the study group, which was not observed within TA muscles. Controls showed significantly higher active $\mathrm{MMO}$ values compared to the study group before CT ( $50.42 \mathrm{~mm}$ vs. $46.31 \mathrm{~mm}, p=0.024)$. The differences between the study group after CT and controls, as well as among the study group before and after $\mathrm{CT}$ did not reach the assumed level of significance in terms of active MMO. The compression technique appears to be effective in the improvement of the active maximal mouth opening and gives significant acute effects on bioelectric masticatory muscle activity. Therefore, CT seems to be effective in MTrPs rehabilitation within the stomatognathic system.
\end{abstract}

Keywords: musculoskeletal rehabilitation; masticatory muscles; surface electromyography; trigger points; masseter muscle; temporal muscle

\section{Introduction}

Masticatory muscle pain is the most common source of pain related to temporomandibular disorders (TMDs), affecting up to $10 \%$ of the population [1]. This medical condition, which is 1.5-2 times 
more frequent in women than in men, can be classified into certain forms such as myofascial pain (MFP), local muscle soreness, protective co-contraction, centrally mediated myalgia, and myospasm [2]. MFP can be caused by myofascial trigger points (MTrPs) which are classified as active or latent [3]. Active MTrPs are associated with spontaneous pain in the surrounding tissue or to distant sites, in comparison to latent MTrPs, which are not associated with a spontaneous pain complaint [4]. Even though elastography, Doppler imaging, diagnostic ultrasound, or biomarkers can be used in the diagnosis of MTrPs, the current gold standard for the MTrPs diagnosis is the manual palpation according to criteria set by Travell and Simons, such as taut band, tender nodule on the taut band with increased pain on pressure, referred recognition pain, and local twitch response [4-6].

Active MTrPs can be associated with a reduced range of motion through muscle dysfunction and muscle weakness. Within the stomatognathic system, MTrPs in the masticatory muscles may restrict active maximum mouth opening (MMO) [7]. Moreover, masticatory muscle pain may contribute to the reduction of maximum bite force [8]. Changes in the masticatory muscles associated with the presence of MTrPs can be observed in the electromyographic examination. MFP among masticatory muscles may be associated with increased surface electromyography (sEMG) values during resting mandibular position [9]. Furthermore, a decrease in bioelectrical masticatory muscle activity during teeth clenching has been demonstrated in patients with MFP within the stomatognathic system [10]. Moreover, the relationship between the presence of MTrPs in masticatory muscles and temporomandibular joint hypomobility is frequently recognized by dentists and physiotherapists.

There are multiple ways to deactivate active MTrPs, including manual therapy, dry needling, local heat/cold, kinesiotaping, ultrasound therapy, and laser therapy [11-13]. Among the manual treatments for MTrPs and MFP, the compression technique (CT), myofascial release, acupressure, muscle energy technique, and specific soft tissue mobilization techniques are most often used. A systematic review and meta-analysis of randomized controlled trials reported that manual MTrPs treatment of head and neck muscles may reduce the frequency, intensity, and duration of tension-type headache, but the quality of evidence was very low [14]. Moreover, it is currently impossible to identify the most effective treatment technique for MTrPs among those proposed. Hence, studies on the effectiveness of manual techniques in the treatment of active MTrPs are necessary.

Although the etiology and pathomechanism of MTrPs among masticatory muscles is complex and not yet fully understood, both diagnosis and treatment of MTrPs is an extremely important clinical aspect in preventing the development of MFP within the stomatognathic system. Thus, the presented study aims to analyze the acute effect of the CT on active maximal mouth opening and the electromyographic activity of the masseter (MM) and temporalis anterior (TA) muscles in subjects with active MTrPs in the MM muscles. The null hypothesis is that CT influences both MMO and bioelectrical masticatory muscle activity in patients with MTrPs. Through the deactivation of active MTrPs, the parameters of resting bioelectrical activity will decrease with an increase in functional activity during teeth clenching.

\section{Materials and Methods}

The presented study was conducted in two stages. In the first stage, people were classified into the study and control groups based on a two-axis Research Diagnostic Criteria for Temporomandibular Disorders (RDC/TMD) and the MTrPs diagnostic criteria presented by Simons et al. [6]. In the second stage, the electromyographic examination and the compression technique within the study group were carried out. This study was carried out at the Department of Functional Masticatory Disorders, Medical University of Lublin, by experienced researchers, dentists, and physiotherapists, in the period between December 2017 and November 2018. The tests were carried out according to the recommendations of the Helsinki Declaration and with the consent of the Bioethics Committee of the Medical University of Lublin (KE-0254/73/2017). All participants were informed about the aim of the study and have given written consent for the research. During qualifying for the research, all participants were clinically examined based on a two-axis Research Diagnostic Criteria for Temporomandibular 
Disorders (RDC/TMD) form by an experienced dentist with a specialization in dental prosthetics (the author M.B. (Magdalena Bakalczuk)). The inclusion criteria used in the study were: female gender, age range 18-23 years, and good or very good general health status according to the RDC/TMD questionnaire, the presence of bilateral active MTrPs in the MM muscles according to diagnostic criteria presented by Simons et al. (study group), and absence of active and/or latent MTrPs in the MM muscles (controls) [6]. The following exclusion criteria were used: the occurrence of headache and cervical spine pain within the month preceding the examination; head and neck injuries within the last six months before the examination; previous head and neck surgical treatment within the last six months before the examination; pregnancy; craniofacial trauma; class II and III of the bite according to Angle's classification; open bite; lack of four support zones in dental arches; lack of more than four teeth within both dental arches; any form of TMDs found according to the RDC/TMD (excluding MM pain in the study group); condition during orthodontic treatment; possession of dental prostheses (regardless of type); mental and neurological disorders. After applying the above criteria, the study group comprised 26 women (mean age $22 \pm 2$ ) with bilateral active MTrPs in the MM muscles. The control group comprised 26 healthy women (mean age $22 \pm 1$ ) without the presence of MTrPs in the MM. The diagnosis of MTrPs was performed by an experienced physiotherapist skilled in the diagnosis of MTrPs (the author M.G.).

Firstly, sEMG of the MM and TA muscles was performed by using the 8-channel electromyograph BioEMG III, compatible with BioPAK Measurement System (BioResearch Associates, Inc. Milwaukee, WI, USA). The sEMG examinations were conducted between 8 and 12 a.m., to minimize the influence of daily fluctuations of muscle activity. During sEMG measurement, the examined person was instructed to sit upright on a dental chair with the head supported, the trunk perpendicular to the floor, the lower limbs straightened, and hands resting on the lap. Before electrode placement, the skin was cleansed with a $90 \%$ ethyl alcohol solution to reduce electrode-skin impedance. The arrangement of surface electrodes was carried out by the same physiotherapist (the author M.G.), following the guidelines of the SENIAM (surface EMG for a non-invasive assessment of muscles) standards [15]. Surface electrodes $\mathrm{Ag} / \mathrm{AgCl}$ with a diameter of $30 \mathrm{~mm}$ and a conductive surface of $16 \mathrm{~mm}$ (SORIMEX, Torun, Poland) were placed in accordance with the course of the muscle fibers of MM and TA muscles, according to the placement technique described by Ferrario and Sforza [16]. The reference electrode was placed on the forehead. Masticatory muscle activity was recorded in two conditions: during resting mandibular position and during maximum voluntary clenching, according to the protocol described by Wieczorek et al. [17]. The electromyographic signals were amplified and purified from $99 \%$ of the noise scale on a linear scale using the BioPak digital NoiseBuster filter. The processing of the sEMG signal by the root mean square (RMS) calculation allowed obtaining average measurement values, which were used to analyze muscle activity.

After the sEMG examination, CT of the active MTrPs in the MM muscles was carried out by the same physiotherapist (the author M.G.). CT was performed bilaterally by pressing with a digital algometer (FDIX, Wagner Instruments, Greenwich, CT, USA) using a constant, calibrated pressure of $2 \mathrm{~kg} / \mathrm{cm}^{2}$ on the MTrPs for $90 \mathrm{~s}$ (Figure 1). Five minutes after CT, the sEMG activity was re-examined according to the same protocol. The active MMO was carried out both before and after CT (the author M.B. (Magdalena Bakalczuk)) Subjects were asked to open mouth as wide as possible without causing an increase in pain. At the end position of maximum mouth opening, the distance between the upper and lower central incisors was measured, according to the protocol described by Ibáñez-García et al. [18].

The data comparison was performed using the IBM SPSS STATISTICS 21 program (IBM Corp., Armonk, NY, USA). First, the normality of the distribution of variables was verified using the Shapiro-Wilk test and the Kolmogorov-Smirnov test (with the Lillierfors correction). All distributions did not fulfill normal distribution, which is why the non-parametric Mann-Whitney U test was used later. The level of significance was determined at $5 \%(p<0.05)$. 


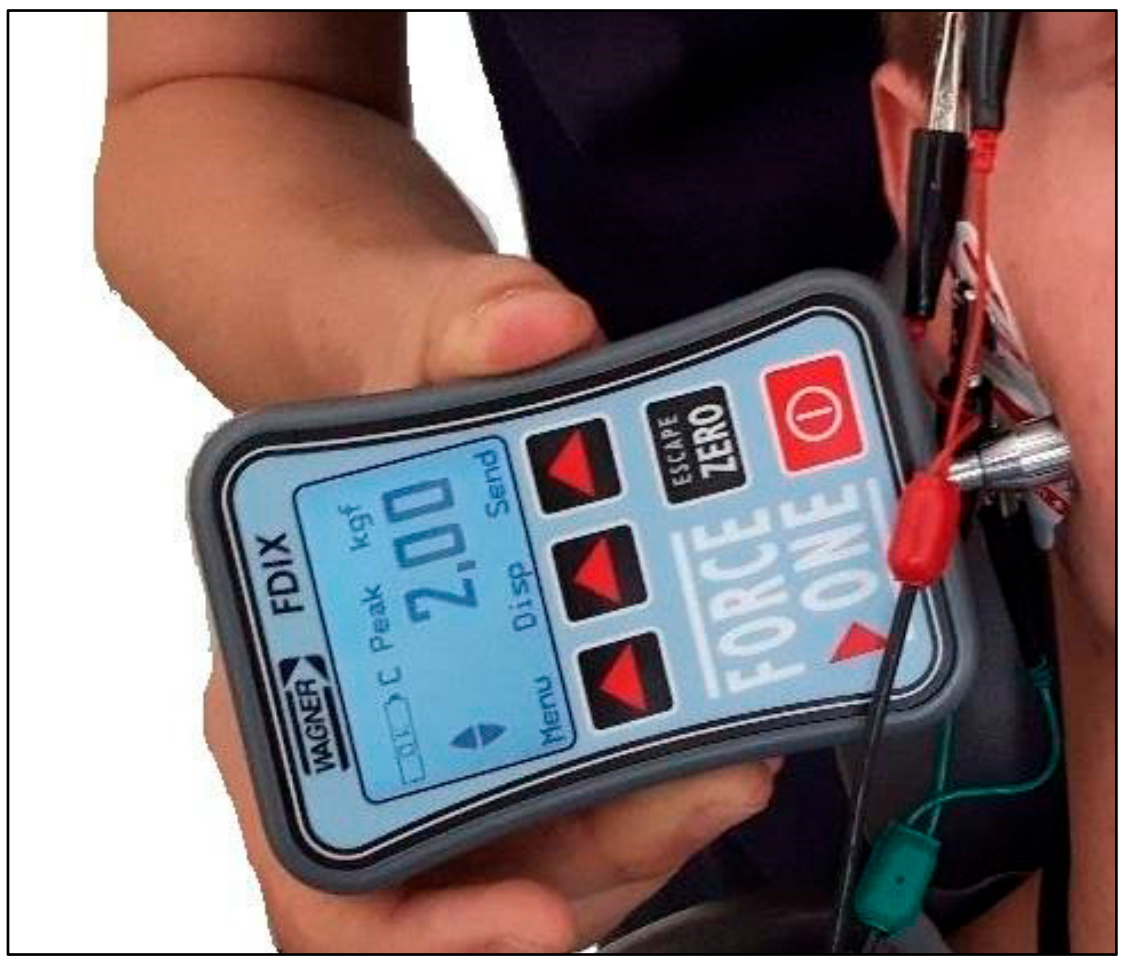

Figure 1. Image of a compression technique over the myofascial trigger point in the masseter muscle performed by pressing with a digital algometer (FDIX, Wagner Instruments, Greenwich, CT, USA).

\section{Results}

There were no significant differences in age $(p=0.415)$ between the study group and controls. Controls showed significantly higher active MMO values compared to the study group before CT (50.42 mm vs. $46.31 \mathrm{~mm}, p=0.024$ ). The differences between the study group after CT and controls, as well as among the study group before and after CT did not reach the assumed level of significance in terms of active MMO ( $p=0.264$ and $p=0.116$, respectively) (Table 1$)$.

Table 1. Comparing means of active maximal mouth opening (MMO).

\begin{tabular}{|c|c|c|c|}
\hline & S1 & S2 & $\mathrm{C}$ \\
\hline $\mathrm{MMO}(\mathrm{mm})$ & 46.31 & 48.46 & 50.42 \\
\hline $\mathrm{SD}(\mathrm{mm})$ & 5.19 & 4.82 & 6.99 \\
\hline $\mathrm{U}$ (S1 vs. C) & 214 & NA & 214 \\
\hline Z (S1 vs. C) & 2.251 & NA & 2.251 \\
\hline p (S1 vs. C) & 0.024 * & NA & 0.024 * \\
\hline r (S1 vs. C) & 0.312 & NA & 0.312 \\
\hline U (S2 vs. C) & NA & & \\
\hline Z (S2 vs. C) & NA & & \\
\hline p (S2 vs. C) & NA & & \\
\hline r (S2 vs. C) & NA & & \\
\hline U (S1 vs. S2) & & & NA \\
\hline Z (S1 vs. S2) & & & NA \\
\hline p (S1 vs. S2) & & & NA \\
\hline r (S1 vs. S2) & & & NA \\
\hline
\end{tabular}

(S1)—study group before compression technique; (S2)—study group after compression technique; (C)—control group; (r) —effect size; * Significant difference.

Table 2 demonstrates the differences between the three groups (the study group before CT, study group after $\mathrm{CT}$, and controls) in resting bioelectric activity. Controls showed significantly lower 
RMS values compared to the study group concerning the mean bioelectrical activity of the TA muscles both before $(1.72 \mu \mathrm{V}$ vs. $3.24 \mu \mathrm{V}, p=0.001)$ and after $\mathrm{CT}(1.72 \mu \mathrm{V}$ vs. $3.2 \mu \mathrm{V}, p=0.001)$. Moreover, controls showed significantly lower RMS values compared to the study group before (1.78 $\mu \mathrm{V}$ vs. $3.09 \mu \mathrm{V}, p=0.001)$ and after $\mathrm{CT}(1.78 \mu \mathrm{V}$ vs. $2.37 \mu \mathrm{V}, p=0.001)$ within the mean bioelectrical activity of the MM muscles. Within the study group, a significant decrease in RMS values was demonstrated concerning the mean bioelectrical activity of the MM muscles after the application of CT (3.09 $\mu \mathrm{V}$ vs. $2.37 \mu \mathrm{V}, p=0.006)$. CT did not affect the resting bioelectrical activity in the TA muscles $(p=0.971)$.

Table 2. Comparing means of bioelectric activity of temporalis anterior (root mean square (RMS) TA) and masseter muscle (RMS MM) during resting mandibular position.

\begin{tabular}{|c|c|c|c|c|}
\hline & S1 & S2 & & $\mathrm{C}$ \\
\hline RMS TA $(\mu V)$ & 3.24 & 3.2 & & 1.72 \\
\hline $\mathrm{SD}(\mu \mathrm{V})$ & 0.77 & 0.62 & & 0.55 \\
\hline U (S1 vs. C) & 39 & NA & & 30 \\
\hline Z (S1 vs. C) & -5.463 & NA & & -5.463 \\
\hline p (S1 vs. C) & 0.001 * & NA & & 0.001 * \\
\hline r (S1 vs. C) & -0.758 & NA & & -0.758 \\
\hline $\mathrm{U}(\mathrm{S} 2$ vs. C) & NA & & 28 & \\
\hline Z (S2 vs. C) & NA & & -5.664 & \\
\hline $\mathrm{p}(\mathrm{S} 2$ vs. $\mathrm{C})$ & NA & & 0.001 * & \\
\hline r (S2 vs. C) & NA & & -0.786 & \\
\hline U (S1 vs. S2) & & & & NA \\
\hline Z (S1 vs. S2) & & & & NA \\
\hline p (S1 vs. S2) & & & & NA \\
\hline r (S1 vs. S2) & & & & NA \\
\hline RMS MM $(\mu \mathrm{V})$ & 3.09 & 2.37 & & 1.78 \\
\hline $\mathrm{SD}(\mu \mathrm{V})$ & 1.09 & 0.76 & & 0.58 \\
\hline $\mathrm{U}$ (S1 vs. C) & 78.5 & NA & & 78.5 \\
\hline Z (S1 vs. C) & -4.74 & NA & & -4.74 \\
\hline p (S1 vs. C) & 0.001 * & NA & & 0.001 * \\
\hline r (S1 vs. C) & -0.657 & NA & & -0.657 \\
\hline U (S2 vs. C) & NA & & 141 & \\
\hline Z (S2 vs. C) & NA & & -3.596 & \\
\hline $\mathrm{p}(\mathrm{S} 2$ vs. C) & NA & & 0.001 * & \\
\hline r (S2 vs. C) & NA & & -0.5 & \\
\hline U (S1 vs. S2) & & & & NA \\
\hline Z (S1 vs. S2) & & & & NA \\
\hline p (S1 vs. S2) & & & & NA \\
\hline r (S1 vs. S2) & & & & NA \\
\hline
\end{tabular}

(S1)—study group before compression technique; (S2)—study group after compression technique; (C)—control group; $(\mathrm{r})$ - effect size; * Significant difference.

Table 3 compares functional masticatory muscle activity during maximum voluntary clenching between the three groups. Controls achieved significantly higher RMS values in TA muscles compared to study group before $(139.06 \mu \mathrm{V}$ vs. $109.91 \mu \mathrm{V}, p=0.022)$ and after $\mathrm{CT}(139.06 \mu \mathrm{V}$ vs. $103.23 \mu \mathrm{V}$, $p=0.03)$. MM muscle activity in the control group was significantly higher only in comparison to the study group after CT $(153.98 \mu \mathrm{V}$ vs. $139.06 \mu \mathrm{V}, p=0.01)$. Within the study group, a significant increase in functional RMS values was demonstrated after the application of CT concerning the mean bioelectrical activity of the MM muscles $(110.20 \mu \mathrm{V}$ vs. $139.06 \mu \mathrm{V}, p=0.014)$, but not TA muscles $(p=0.577)$. 
Table 3. Comparing means of bioelectric activity of temporalis anterior (RMS TA) and masseter muscle (RMS MM) during maximum voluntary clenching.

\begin{tabular}{|c|c|c|c|c|}
\hline & S1 & S2 & & $\mathrm{C}$ \\
\hline RMS TA $(\mu \mathrm{V})$ & 109.91 & 103.23 & & 139.06 \\
\hline $\mathrm{SD}(\mu \mathrm{V})$ & 52.83 & 42.03 & & 75.38 \\
\hline U (S1 vs. C) & 212 & NA & & 212 \\
\hline Z (S1 vs. C) & 2.297 & NA & & 2.297 \\
\hline p (S1 vs. C) & $0.022 *$ & NA & & $0.022 *$ \\
\hline r (S1 vs. C) & 0.319 & NA & & 0.319 \\
\hline U (S2 vs. C) & NA & & 219 & \\
\hline Z (S2 vs. C) & NA & & -2.169 & \\
\hline p (S2 vs. C) & NA & & $0.03 *$ & \\
\hline r (S2 vs. C) & NA & & -0.301 & \\
\hline U (S1 vs. S2) & & & & NA \\
\hline Z (S1 vs. S2) & & & & NA \\
\hline p (S1 vs. S2) & & & & NA \\
\hline r (S1 vs. S2) & & & & NA \\
\hline RMS MM $(\mu \mathrm{V})$ & 110.2 & 139.06 & & 153.98 \\
\hline $\mathrm{SD}(\mu \mathrm{V})$ & 58.38 & 41.44 & & 98.88 \\
\hline U (S1 vs. C) & 255 & NA & & 255 \\
\hline Z (S1 vs. C) & 1.51 & NA & & 1.51 \\
\hline p (S1 vs. C) & 0.131 & NA & & 0.131 \\
\hline r (S1 vs. C) & 0.209 & NA & & 0.209 \\
\hline U (S2 vs. C) & NA & & 197 & \\
\hline Z (S2 vs. C) & NA & & -0.229 & \\
\hline p (S2 vs. C) & NA & & $0.01 *$ & \\
\hline r (S2 vs. C) & NA & & -0.032 & \\
\hline U (S1 vs. S2) & & & & NA \\
\hline Z (S1 vs. S2) & & & & NA \\
\hline p (S1 vs. S2) & & & & NA \\
\hline r (S1 vs. S2) & & & & NA \\
\hline
\end{tabular}

(S1)—study group before compression technique; (S2)—study group after compression technique; (C)—control group; (r) — effect size; * Significant difference.

\section{Discussion}

Among various manual treatments for MFP associated with MTrPs, the most commonly used are compression technique, myofascial release, acupressure, muscle energy technique, and specific soft tissue mobilization techniques [14]. However, manual treatments for MTrPs have varying degrees of effectiveness and it is hard to identify the most effective rehabilitation method for MTrPs.

The presented study aims to analyze the acute effect of the compression technique on active maximum mouth opening and bioelectric masticatory muscle activity in young adult women with active myofascial trigger points in the masseter muscles. To the best of our knowledge, this is the first study to evaluate the acute effects of CT in masticatory muscles with active MTrPs using sEMG. Although the increase in the active MMO values within the study group after CT was not statistically significant, a significant difference was observed only before CT between the study and control groups. For determination of impact on masticatory muscle activity, surface electromyography was applied, by using a BioEMG III device in resting mandibular position and during maximum voluntary clenching. The main finding of the presented study was that the resting bioelectrical activity of the MM muscles with active MTrPs after CT was significantly lower than before CT. Opposite interaction was found in bioelectric MM muscle activity during maximum voluntary contraction. A significant increase in RMS values during maximum voluntary clenching was demonstrated after the application of CT concerning the bioelectrical activity of the MM muscles. The above observations seem to be in line with the pain adaptation model, hypothesizing that pain leads to changes in muscle activity aiming to limit movement and to protect tissues against further injuries by a decreased muscle activity [19]. The above 
hypothesis is in accordance with the results presented by Manfredini et al. who showed significantly higher masticatory muscle activity during clenching tasks in TMDs-free subjects in comparison to patients diagnosed with TMDs [10]. Moreover, Pietropaoli et al. reported higher resting bioelectrical activity of masticatory muscles in patients with myofascial pain [9]. The pain adaptation model seems to be also confirmed by the presented observations, which show alterations in sEMG activity due to the presence of MTrPs. Hence, the deactivation of MTrPs through CT improves muscle activity and offers the possibility of generating greater forces within the MM muscles.

Both the increase in active MMO and changes in SEMG activity after CT may have a multifactorial basis. Local pressure may equalize the length of sarcomeres in the involved MTrPs, which would help to dictate the length and tone into the affected tissues $[18,20]$. On the other hand, changes in MMO after CT may result from reactive hyperemia in the MTrPs region or a spinal reflex mechanism for the relief of muscle spasm [21]. Although the mechanism of CT has not been clearly explained, the therapeutic effect has been confirmed in several studies [22-28]. In the study of Aguilera et al., the decrease in bioelectrical activity after MTrPs compression was related to the improvement of active range of motion, which was also confirmed in the presented work [22]. The study of Kisilewicz et al. confirms that a single treatment session of active MTrPs with CT causes a decrease in muscle stiffness [25]. Moreover, Cagnie et al. observed that a 4-week treatment of MTrPs for CT resulted in a significant improvement in pressure pain sensitivity, mobility, and muscle strength in the short term [26]. On the other hand, the long-term impact of CT on improvement in functional capacities after the 15 treatments was observed by Hains et al. [27]. Based on the systematic review of Cagnie et al., CT can be recommended in the treatment of neck pain patients with MTrPs [28]. However, current literature lacks research on the CT application in patients with active MTrPs within the stomatognathic system.

Within the present study, we provided an objective confirmation of the efficacy of $\mathrm{CT}$ from a clinical perspective. Based on the obtained results, CT seems to be effective in the improvement of the active maximal mouth opening and gives significant acute effects on bioelectric masticatory muscle activity. Therefore, a practical application of this study is that CT influencing the functional and resting activity of the masticatory muscles may be useful in the treatment of MTrPs within the stomatognathic system. Thus, future clinical trials are needed to confirm the effectiveness of CT application within masticatory muscles as well as to assess whether it is more effective compared to other techniques such as myofascial release, acupressure, muscle energy technique, or dry needling. In addition, the described procedure can be used as a standardized test protocol for assessing the effectiveness of therapy within the masticatory system. The presented study has several limitations. Firstly, the diagnostics criteria for TMDs were replaced by DC/TMDs in 2014. However, in this study, the previous version was used. There is no validated Polish version of the DC/TMDs so far, therefore, the RDC/TMDs was used. Secondly, the study sample consists of young adult women. We decided to include only women in the presented research because masticatory muscle pain associated with MTrPs is 1.5-2 times more frequent in women than in men. Thus, future research should include the male population with an expanded age range. Thirdly, a generalization of the findings is limited by the short-term follow-up used in the presented study. Therefore, a longer period of follow-up is recommended to determine the long-term effects of the compression technique in patients with masticatory muscle pain. Moreover, in a heterogeneous population of healthy adults, natural variations in age, height, and weight significantly affect the MMO [29]. In the presented study, there were no significant differences in age between the study group and controls. However, weight and height were not included in the comparison. Hence, future research should also include demographic data in this area.

\section{Conclusions}

The compression technique appears to be effective in the improvement of the active maximal mouth opening and gives significant acute effects on bioelectric masticatory muscle activity. Therefore, $\mathrm{CT}$ seems to be effective in MTrPs rehabilitation within the stomatognathic system. Future clinical 
trials are needed to observe the long-term impact and effectiveness of CT application in patients with masticatory muscle pain.

Author Contributions: Conceptualization, M.G. and M.B. (Marcin Berger); methodology, M.G. and M.B. (Marcin Berger); software, M.G.; formal analysis, G.Z.; investigation, M.B. (Magdalena Bakalczuk) and M.G.; resources, J.S.; data curation, G.Z.; writing-original draft preparation, M.G. and G.Z.; writing-review and editing, M.G.; visualization, M.G.; supervision, P.M. and M.G.; project administration, J.S.; funding acquisition, J.S. and P.M. All authors have read and agreed to the published version of the manuscript.

Funding: This research received no external funding.

Conflicts of Interest: The authors declare no conflict of interest.

\section{References}

1. Manfredini, D.; Guarda-Nardini, L.; Winocur, E.; Piccotti, F.; Ahlberg, J.; Lobbezoo, F. Research diagnostic criteria for temporomandibular disorders: A systematic review of axis I epidemiologic findings. Oral Surgery Oral Med. Oral Pathol. Oral Radiol. Endodontology 2011, 112, 453-462. [CrossRef] [PubMed]

2. Wieckiewicz, M.; Zietek, M.; Smardz, J.; Zenczak-Wieckiewicz, D.; Grychowska, N. Mental Status as a Common Factor for Masticatory Muscle Pain: A Systematic Review. Front. Psychol. 2017, 8, 8. [CrossRef] [PubMed]

3. Jafri, M.S. Mechanisms of Myofascial Pain. Int. Sch. Res. Not. 2014, 2014, 1-16. [CrossRef] [PubMed]

4. Shah, J.P.; Thaker, N.; Ba, J.H.; Bs, J.V.A.; Sikdar, S.; Gerber, L.H. Myofascial Trigger Points Then and Now: A Historical and Scientific Perspective. PMER 2015, 7, 746-761. [CrossRef]

5. Srbely, J.Z.; Kumbhare, D.; Grosman-Rimon, L. A narrative review of new trends in the diagnosis of myofascial trigger points: Diagnostic ultrasound imaging and biomarkers. J. Can. Chiropr. Assoc. 2016, 60, 220-225.

6. Simons, D.G.; Travell, J.G.; Simons, L.S. Travell E Simons' Myofascial Pain and Dysfunction: The Trigger Point Manual, 2nd ed.; Williams \& Wilkins: Baltimore, MD, USA, 1999; pp. 329-365.

7. De-La-Llave-Rincón, A.I.; Alonso-Blanco, C.; Gil-Crujera, A.; Ambite-Quesada, S.; Svensson, P.; Fernández-De-Las-Peñas, C. Myofascial Trigger Points in the Masticatory Muscles in Patients With and Without Chronic Mechanical Neck Pain. J. Manip. Physiol. Ther. 2012, 35, 678-684. [CrossRef]

8. Koc, D.; Dogan, A.; Bek, B. Bite Force and Influential Factors on Bite Force Measurements: A Literature Review. Eur. J. Dent. 2010, 4, 223-232. [CrossRef]

9. Pietropaoli, D.; Ortu, E.; Giannoni, M.; Cattaneo, R.; Mummolo, A.; Monaco, A. Alterations in Surface Electromyography Are Associated with Subjective Masticatory Muscle Pain. Pain Res. Manag. 2019, 2019, 1-9. [CrossRef]

10. Manfredini, D.; Cocilovo, F.; Favero, L.; Ferronato, G.; Tonello, S.; Guarda-Nardini, L. Surface electromyography of jaw muscles and kinesiographic recordings: Diagnostic accuracy for myofascial pain. J. Oral Rehabil. 2011, 38, 791-799. [CrossRef]

11. Yildirim, M.A.; Yildirim, M.A.; Öneş, K.; Gökşenoğlu, G. Effectiveness of Ultrasound Therapy on Myofascial Pain Syndrome of the Upper Trapezius: Randomized, Single-Blind, Placebo-Controlled Study. Arch. Rheumatol. 2018, 33, 418-423. [CrossRef]

12. Majlesi, J.; Ünalan, H. Effect of Treatment on Trigger Points. Curr. Pain Headache Rep. 2010, 14, $353-360$. [CrossRef]

13. Kalichman, L.; Levin, I.; Bachar, I.; Vered, E. Short-term effects of kinesio taping on trigger points in upper trapezius and gastrocnemius muscles. J. Bodyw. Mov. Ther. 2017, 22, 700-706. [CrossRef] [PubMed]

14. Maistrello, L.F.; Geri, T.; Gianola, S.; Zaninetti, M.; Testa, M. Effectiveness of Trigger Point Manual Treatment on the Frequency, Intensity, and Duration of Attacks in Primary Headaches: A Systematic Review and Meta-Analysis of Randomized Controlled Trials. Front. Neurol. 2018, 9, 254. [CrossRef]

15. Hermens, H.J.; Freriks, B.; Disselhorst-Klug, C.; Rau, G. Development of recommendations for SEMG sensors and sensor placement procedures. J. Electromyogr. Kinesiol. 2000, 10, 361-374. [CrossRef]

16. Ferrario, V.F.; Sforza, C. Coordinated electromyographic activity of the human masseter and temporalis anterior muscles during mastication. Eur. J. Oral Sci. 1996, 104, 511-517. [CrossRef] 
17. Wieczorek, A.; Loster, J.; Loster, B.W. Relationship between Occlusal Force Distribution and the Activity of Masseter and Anterior Temporalis Muscles in Asymptomatic Young Adults. BioMed Res. Int. 2012, 2013, 1-7. [CrossRef]

18. Ibáñez-García, J.; Alburquerque-Sendín, F.; Rodríguez-Blanco, C.; Girao, D.; Atienza-Meseguer, A.; Planella-Abella, S.; Peñas, C.F.-D.-L. Changes in masseter muscle trigger points following strain-counterstrain or neuro-muscular technique. J. Bodyw. Mov. Ther. 2009, 13, 2-10. [CrossRef] [PubMed]

19. Murray, G.M.; Peck, C.C. Orofacial pain and jaw muscle activity: A new model. J. Orofac. Pain 2007, 21, 263-278.

20. Simons, D.G. Understanding effective treatments of myofascial trigger points. J. Bodyw. Mov. Ther. 2002, 6, 81-88. [CrossRef]

21. Hou, C.-R.; Tsai, L.-C.; Cheng, K.-F.; Chung, K.-C.; Hong, C.-Z. Immediate effects of various physical therapeutic modalities on cervical myofascial pain and trigger-point sensitivity. Arch. Phys. Med. Rehabil. 2002, 83, 1406-1414. [CrossRef]

22. Aguilera, F.J.M.; Pecos-Martín, D.; Masanet, R.A.; Botella, A.C.; Soler, L.B.; Morell, F.B. Immediate Effect of Ultrasound and Ischemic Compression Techniques for the Treatment of Trapezius Latent Myofascial Trigger Points in Healthy Subjects: A Randomized Controlled Study. J. Manip. Physiol. Ther. 2009, 32, 515-520. [CrossRef]

23. Kostopoulos, D.; Nelson, A.J., Jr.; Ingber, R.S.; Larkin, R.W. Reduction of Spontaneous Electrical Activity and Pain Perception of Trigger Points in the Upper Trapezius Muscle through Trigger Point Compression and Passive Stretching. J. Musculoskelet. Pain 2008, 16, 266-278. [CrossRef]

24. Lietz-Kijak, D.; Kopacz, Ł.; Ardan, R.; Grzegocka, M.; Kijak, E. Assessment of the Short-Term Effectiveness of Kinesiotaping and Trigger Points Release Used in Functional Disorders of the Masticatory Muscles. Pain Res. Manag. 2018, 2018, 1-7. [CrossRef]

25. Kisilewicz, A.; Janusiak, M.; Szafraniec, R.; Smoter, M.; Ciszek, B.; Madeleine, P.; Fernández-De-Las-Peñas, C.; Kawczyński, A. Changes in Muscle Stiffness of the Trapezius Muscle after Application of Ischemic Compression into Myofascial Trigger Points in Professional Basketball Players. J. Hum. Kinet. 2018, 64, 35-45. [CrossRef]

26. Cagnie, B.; Dewitte, V.; Coppieters, I.; Van Oosterwijck, J.; Cools, A.; Danneels, L. Effect of Ischemic Compression on Trigger Points in the Neck and Shoulder Muscles in Office Workers: A Cohort Study. J. Manip. Physiol. Ther. 2013, 36, 482-489. [CrossRef] [PubMed]

27. Hains, G.; Descarreaux, M.; Lamy, A.-M.; Hains, F. A randomized controlled (intervention) trial of ischemic compression therapy for chronic carpal tunnel syndrome. J. Can. Chiropr. Assoc. 2010, 54, 155-163. [PubMed]

28. Cagnie, B.; Castelein, B.; Pollie, F.; Steelant, L.; Verhoeyen, H.; Cools, A. Evidence for the Use of Ischemic Compression and Dry Needling in the Management of Trigger Points of the Upper Trapezius in Patients with Neck Pain. Am. J. Phys. Med. Rehabil. 2015, 94, 573-583. [CrossRef]

29. Venkatraman, A.; Kaval, F.; Takiar, V. Body Mass Index and Age Affect Maximum Mouth Opening in a Contemporary American Population. J. Oral Maxillofac. Surg. 2020, 78, 1926-1932. [CrossRef]

Publisher's Note: MDPI stays neutral with regard to jurisdictional claims in published maps and institutional affiliations.

(C) 2020 by the authors. Licensee MDPI, Basel, Switzerland. This article is an open access article distributed under the terms and conditions of the Creative Commons Attribution (CC BY) license (http://creativecommons.org/licenses/by/4.0/). 